\title{
An Evaluation of LES Turbulence Models for Scalar Mixing in the Stratocumulus-Capped Boundary Layer
}

\author{
Xiaoming Shi And Fotini Katopodes Chow \\ Department of Civil and Environmental Engineering, University of California, Berkeley, Berkeley, California \\ ROBERT L. STREET \\ Department of Civil and Environmental Engineering, Stanford University, Stanford, California \\ GEORGE H. BRYAN \\ National Center for Atmospheric Research, Boulder, Colorado
}

(Manuscript received 23 December 2017, in final form 15 March 2018)

\begin{abstract}
The stratocumulus cloud-capped boundary layer under a sharp inversion is a challenging regime for largeeddy simulation (LES). Here, data from the first research flight of the Second Dynamics and Chemistry of the Marine Stratocumulus field study are used to evaluate the effect of different LES turbulence closures. Six different turbulence models, including traditional TKE and Smagorinsky models and more advanced models that employ explicit filtering and reconstruction, are tested. The traditional models produce unrealistically thin clouds and a decoupled boundary layer as compared with other more advanced models. Traditional models rely on specified subfilter-scale (SFS) Prandtl and Schmidt numbers to obtain SFS eddy diffusivity from eddy viscosity, whereas dynamic models can compute SFS eddy diffusivity independently through dynamic procedures. The effective SFS Prandtl number in dynamic models is found to be $\sim 0.5$ below the cloud and $\sim 10$ inside the cloud layer, implying minimized mixing in the cloud. In contrast, the SFS Prandtl number in traditional models is about $1 / 3$ throughout the entire boundary layer, suggesting spuriously strong mixing in the cloud. The SFS Schmidt number in the dynamic models also changes independently from the SFS Prandtl number, whereas in traditional models they are identical, meaning that the efficiency of the turbulent mixing of water content is forced to be the same as that of heat. Since it is very difficult to know in advance the SFS Prandtl and Schmidt numbers in a given flow, dynamic models may provide a more realistic representation of scalar mixing in LES.
\end{abstract}

\section{Introduction}

Large-eddy simulation (LES), which resolves the energetic eddies responsible for most of the transport of momentum, energy, and mass through the boundary layer, has been an essential tool for developing boundary layer theory and parameterizations (e.g., Vanzanten et al. 1999; Moeng 2000; Stevens et al. 2005). When LES is applied to stratocumulus clouds with sharp temperature inversions, however, many models produce an unrealistically thin cloud layer and a decoupled boundary layer (Stevens et al. 2005).

The choice of turbulence closure model for the stratocumulus-capped boundary layer (SCBL) has been

Corresponding author: Xiaoming Shi, shixm@berkeley.edu examined by Stevens et al. (2005), Kirkpatrick et al. (2006), Matheou and Chung (2014), and Shi et al. (2018), but relatively little attention has been given to the choice of the Prandtl number (Pr) and the Schmidt number (Sc) for subfilter-scale (SFS) scalar diffusivities. Shi et al. (2018) used explicit filtering and reconstruction for simulations of the stratocumulus clouds and found improved performance in terms of maintaining cloud water and boundary layer coupling in their simulations. Kirkpatrick et al. (2006) and Stevens et al. (2005) also showed that a dynamic model yields similar improvements. In the dynamic approach, SFS eddy diffusivities for scalar mixing are computed independently, in contrast to traditional models, which obtain SFS eddy diffusivities from eddy viscosities with empirically determined $\mathrm{Pr}$ and Sc. There is considerable literature devoted to 
determining these numbers (e.g., Venayagamoorthy and Stretch 2010; Wilson and Venayagamoorthy 2015; Goldberg et al. 2010), and, according to Goldberg et al. (2010), both Pr and Sc vary within individual flows so that the employment of constant values throughout a flow or even a time-constant empirical construct is not adequate.

In this paper, we directly investigate turbulent scalar fluxes and the role of Pr and Sc in simulations of the SCBL by examining six model variations: traditional formulations [turbulent kinetic energy (TKE) and Smagorinsky model (SM)], fully dynamic formulations [dynamic Wong-Lilly (DWL-D) and dynamic reconstruction model (DRM-D)], and dynamic formulations using an empirical specification of the Prandtl and Schmidt numbers [DWL with a Prandtl number (DWL-Pr) and DRM with a Prandtl number (DRM-Pr)]. In the latter two cases, the dynamic procedure is only applied to the momentum equations. Applying the dynamic procedure to the eddy diffusivities separately is a significant departure from traditional formulations, and its consequences and benefits are the focus of this work. We compare our simulation results with data from the first research flight (RF01) of the Second Dynamics and Chemistry of the Marine Stratocumulus field study (DYCOMS II).

\section{Turbulence closure models}

The LES code used is the Cloud Model 1 (CM1), release 18 , which solves the nonhydrostatic, compressible equations of the moist atmosphere (Bryan and Fritsch 2002). Applying the LES spatial filter (overbar) to these equations yields a set of filtered equations for the velocity components $\bar{u}_{i}$, the potential temperature $\bar{\theta}$, the cloud liquid water mixing ratio $\bar{q}_{c}$, and the water vapor mixing ratio $\bar{q}_{v}$. The unresolvable turbulent stresses and fluxes (for which models are required) are SFS stresses $\tau_{i j}=\overline{u_{i} u_{j}}-\bar{u}_{i} \bar{u}_{j}$, SFS flux of heat $\tau_{\theta j}=\overline{\theta u_{j}}-\bar{\theta} \bar{u}_{j}$, and SFS flux of mixing ratios for water $\tau_{q j}=\overline{q u_{j}}-\bar{q} \bar{u}_{j}$.

In the models employing an SFS eddy viscosity and/or eddy diffusivity, such as the SM, the SFS stress is determined by $\tau_{i j}=-2 K_{m} \bar{S}_{i j}$, where $K_{m}$ is an eddy viscosity and $\bar{S}_{i j}=\left(\partial \bar{u}_{i} / \partial x_{j}+\partial \bar{u}_{j} / \partial x_{i}\right) / 2$ is the resolved strain-rate tensor. Then SFS flux of scalars is determined similarly with an eddy diffusivity $K_{h}$ and a scalar gradient $\partial \bar{\phi} / \partial x_{j}$, where $\phi$ is a scalar. Often, $K_{h}$ is simply given by $K_{h}=K_{m} / \operatorname{Pr}$, where $\operatorname{Pr}=1 / 3$ is the Prandtl number.

SFS stress and fluxes in the 1.5-order TKE scheme (Deardorff 1980) are also based on eddy viscosity and diffusivity. A prognostic equation generates the SFS TKE $(e)$. The SFS eddy viscosity is then proportional to $\sqrt{e}$, a stability-dependent length scale and a selected coefficient. Pr is $1 / 3$ for unstable and neutral conditions and becomes 1 for strongly stable stratification.
In the dynamic model developed by Wong and Lilly (1994) (DWL), $K_{m}$ and $K_{h}$ are computed with a dynamic procedure (DWL-D). Then the eddy diffusivity for total water $\left(\bar{q}_{c}+\bar{q}_{v}\right)$ is also computed dynamically, making it independent of the eddy viscosity calculation.

Alternatively, an empirical expression can be used for the SFS Pr and Sc values. Here, the parameterization of Pr developed by Venayagamoorthy and Stretch (2010) is used:

$$
\operatorname{Pr}=\operatorname{Pr}_{0} \exp \left(-\frac{\mathrm{Ri}}{\operatorname{Pr}_{0} \Gamma_{\infty}}\right)+\frac{\mathrm{Ri}}{\mathrm{Ri}_{f \infty}},
$$

where $\operatorname{Pr}_{0}=0.7, \mathrm{Ri}_{f \infty}=0.25, \Gamma_{\infty}=\mathrm{Ri}_{f \infty} /\left(1-\mathrm{Ri}_{f \infty}\right)=1 / 3$, and $\mathrm{Ri}$ is the Richardson number, defined as the ratio of the square of Brunt-Väisälä frequency and the deformation, Sc is assumed to be the same as Pr, and $K_{h}=K_{m} / \operatorname{Pr}$. This approach saves computation time because only the dynamic procedure for $K_{m}$ is needed and is denoted DWL-Pr. We note that (1) is derived from an unsteady Reynolds-averaged Navier-Stokes (RANS) perspective, but a number of authors have made use of appropriate unsteady RANS parameterizations in the LES context (e.g., Findikakis and Street 1979; Perot and Gadebusch 2009; Rasam et al. 2013). This provides a useful tool to allow us to examine the effect of using the dynamic procedure only on momentum while providing a somewhat more sophisticated Pr model.

When a framework based on explicit filtering is employed, the SFS motions are divided into resolvable subfilter scales (RSFSs) and unresolvable subgrid scales (SGSs). The RSFS stresses can be modeled with reconstructed velocities $\tilde{u}_{i}^{*}$, represented here by the lowestlevel reconstruction $\tilde{u}_{i}^{*}=\tilde{\tilde{u}}_{i}$ [see the appendix of Shi et al. (2018) for a discussion of the effects of higher-order reconstruction], where the tilde denotes the effect of discretization and the overbar denotes the explicit filter. If an eddy viscosity model is used to represent $\tau_{i j}^{\mathrm{SGS}}$, then

$$
\tau_{i j}=\underbrace{-2 K_{m} \bar{S}_{i j}}_{\tau_{i j}^{\mathrm{SGS}}}+\underbrace{\left(\overline{\tilde{\tilde{u}}}_{i} \overline{\tilde{u}}_{j}-\overline{\tilde{\tilde{u}}}_{i} \overline{\tilde{\tilde{u}}}_{j}\right)}_{\tau_{i j}^{\text {RSFS }}} .
$$

If the SGS stress is simply modeled with the Smagorinsky model, (2) becomes the well-known mixed model of Bardina et al. (1983), for which the RSFS term with zerolevel reconstruction is effectively the scale-similarity closure. Similarly, the SFS flux of a scalar becomes, for example, for $\theta$,

$$
\tau_{\theta j}=\underbrace{-K_{h} \frac{\partial \bar{\theta}}{\partial x_{j}}}_{\tau_{\theta j}^{\mathrm{SGS}}}+\underbrace{\left(\overline{\tilde{\tilde{\theta}}} \overline{\tilde{u}}_{j}-\overline{\tilde{\tilde{\theta}}} \overline{\tilde{u}}_{j}\right)}_{\tau_{\theta j}^{\mathrm{RFFS}}} .
$$


In the DRM (Chow et al. 2005), SGS eddy viscosity and the eddy diffusivities can be computed dynamically (DRM-D) or using a specified Prandtl number (DRM-Pr). Thus, similar to having DWL-D and DWL-Pr, for DRM-D, dynamic modeling is applied to SGS fluxes of potential temperature and total water mixing ratio separately, while DRM-Pr uses the Prandtl number in (1) to obtain an eddy diffusivity from the dynamically computed eddy viscosity. The reconstruction procedure of DRM-Pr is the same as DRM-D (i.e., the RSFS term is computed for every variable in the simulations); this is the main reason why DRM-Pr and DRM-D exhibit similar results (see section 3a).

Our simulations follow Stevens et al. (2005). The horizontal grid spacing is $\Delta x=\Delta y=35 \mathrm{~m}$, and the vertical resolution is $\Delta z=5 \mathrm{~m}$. The number of horizontal grid points is $n_{x}=n_{y}=96$, and the vertical extent of the domain is $1.5 \mathrm{~km}$. All simulations were run for $4 \mathrm{~h}$, and after the first hour of spinup, all simulations become statistically steady. A fifth-order weighted essentially nonoscillatory (WENO) scheme (Borges et al. 2008) is used for the advection of momentum and scalars. As discussed in Shi et al. (2018), this case is sensitive to the choice of advection scheme (cf. Pressel et al. 2017), which can add numerical diffusion to the mixing from the turbulence closure. Shi et al. (2018) report that the DRM, for example, consistently provides significant improvement over traditional Smagorinsky and TKE models for all the alternative advection schemes they tested. Microphysics processes are represented with a simple scheme by Bryan and Rotunno (2009). Other details of the turbulence models and simulation setup can be found in Shi et al. (2018).

With the above setup, the normalized computational costs for the simulations using SM, TKE, DWL-Pr, DWL-D, DRM-Pr, and DRM-D are 1.00, 1.01, 1.07, $1.12,1.16$, and 1.20 , respectively, where the actual computation time for the SM run is used as the reference. Notably, DWL-D only increases the computational cost by an additional 5\% relative to DWL-Pr. This is a very small increase considering the resulting improvement, which is discussed below.

\section{Simulations}

\section{a. An intercomparison between turbulence models}

Figure 1 shows profiles of the mixing ratio of cloud water $q_{c}$ for the various turbulence models. Field observations suggested that the cloud retains its initial water profile over time; however, the maxima of $q_{c}$ in SM, TKE, and DWL-Pr are only $1 / 3-1 / 2$ of the initial maximum. DWL-D, DRM-Pr, and DRM-D exhibit significantly larger peak values of $q_{c}$ than others, and

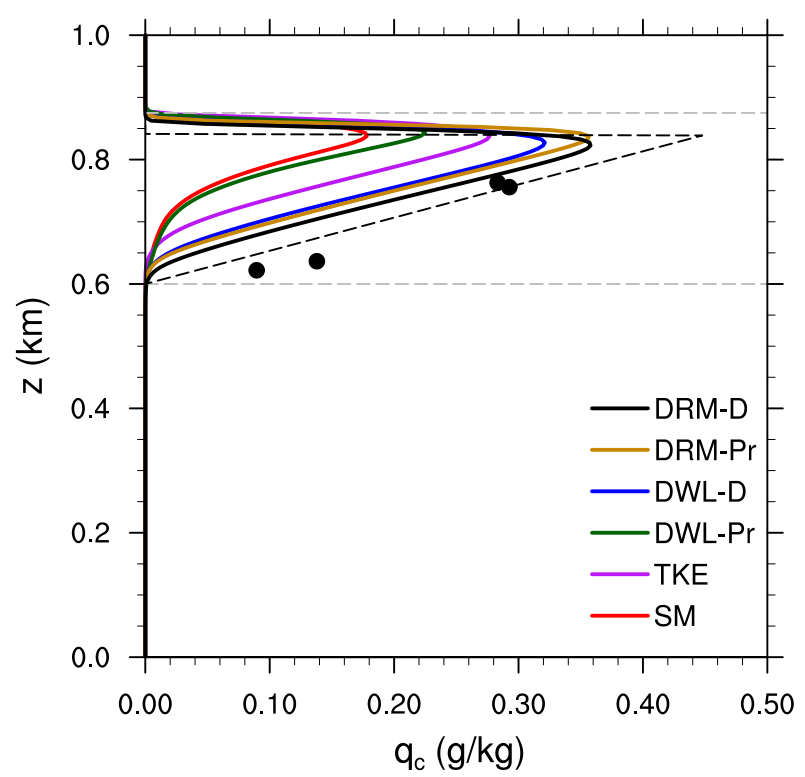

FIG. 1. Horizontal- and time-mean profiles of cloud water mixing ratios $q_{c}$ over the fourth hour of the simulations. The dashed black profile corresponds to $q_{c}$ from the initial conditions. Solid dots indicate the observed values of $q_{c}$ during DYCOMS II RF01 (Stevens et al. 2005). Two dashed gray lines in this and the following figures indicate cloud base and cloud top at the end of the simulations.

their cloud layer is also much thicker. DRM-D maintains the most liquid water among all simulations.

Figure $2 \mathrm{a}$ shows the variance of resolved vertical velocity $w$. The observation data indicate the presence of a single peak in the profile. SM and DWL-Pr show two peaks. TKE shows a single peak with intensity significantly weaker than the observations. In contrast, DWL-D, DRM-Pr, and DRM-D have well-defined single-peak structures and larger variances. The strength of resolved turbulence is strongest in DRM-D, which best matches observation data.

The resolved third central moment of $w$ is shown in Fig. 2b. Negative value indicates the presence of strong downdrafts, as expected in a flow predominantly driven by cloud-top cooling, whereas positive value indicates surface-heating-driven turbulence and cumulus convection. SM and DWL-Pr completely miss the negative skewness in the observation data. The TKE model captures a weak negative peak in the cloud layer, possibly due to keeping more cloud water than SM and DWL-Pr, but it still has a strong positive peak near the surface. DWL-D, DRM-Pr, and DRM-D exhibit a good match with observation data and show significant negative peaks right above cloud base.

Overall, the TKE model and SM fail to produce physically realistic results in the simulations. The DWL-D results are generally better, but the DWL-Pr results are 
a)

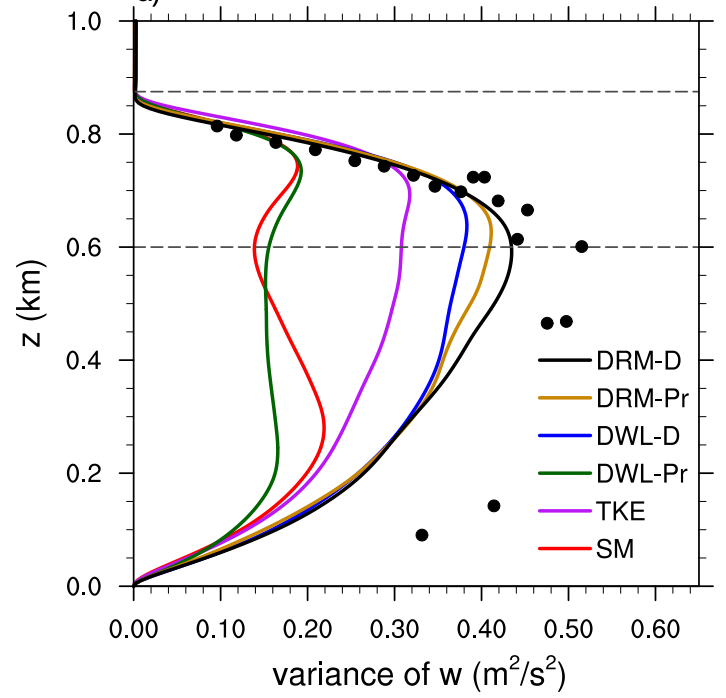

b)

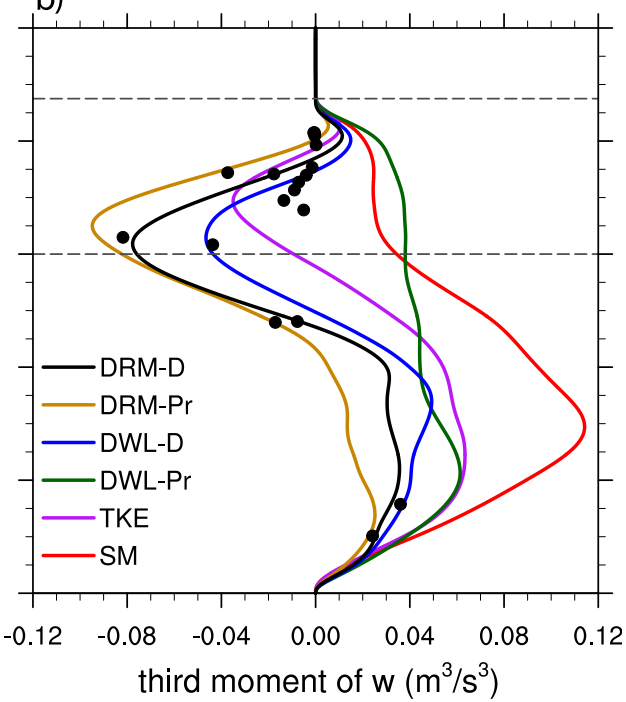

FIG. 2. Horizontal- and time-mean profiles of (a) the variance and (b) the third moment of resolved vertical velocity $w$ over the fourth hour of the simulations. Solid dots correspond to the variance and third moment of $w$ in observation data.

poor, suggesting that accurate modeling of scalar transport is key. The best results are obtained with the reconstruction-based DRM-D, with the DRM-Pr simulation being slightly inferior for the variance and cloud water.

\section{b. Eddy viscosity}

What is perhaps surprising is that the TKE model and SM have lower eddy viscosities than do the other models depicted in Fig. 3. The eddy viscosity for TKE and SM is about $0.15 \mathrm{~m}^{2} \mathrm{~s}^{-1}$ at most levels of the boundary layer, whereas the eddy viscosity for the other models is around $0.6 \mathrm{~m}^{2} \mathrm{~s}^{-1}$ in the boundary layer. All models show values going to zero at cloud top. The contrast within the boundary layer is rooted in the different methodologies for determining eddy viscosities. With SM and TKE, eddy viscosity is parameterized as a function of the strain-rate tensor and the SFS TKE, respectively, though in the TKE scheme, the production of SFS TKE is still mainly controlled by the strain rate. In the dynamic models, eddy viscosity is determined by the dynamic calculation of the ratio of test-filter-level stresses and the strain-rate tensor. Therefore, while the eddy viscosity in the SM and TKE model decreases as the strain rate of the flow decreases, the dynamic models do not necessarily predict the same dependence. For a given strain rate, the eddy viscosity in DWL can be either very large or possibly zero (corresponding to flow regions that exhibit backscatter), depending on specific test-filter-level stresses.

\section{c. Eddy diffusivity}

The eddy diffusivities for potential temperature $K_{h}$ are depicted in Fig. 4a. DRM-D and DWL-D, the two models having separate dynamic procedures for the scalars, show a distinctly different profile from the others, with a sharp transition at the cloud base. Their eddy diffusivities are relatively large below the cloud but very small within the cloud layer, indicating significantly

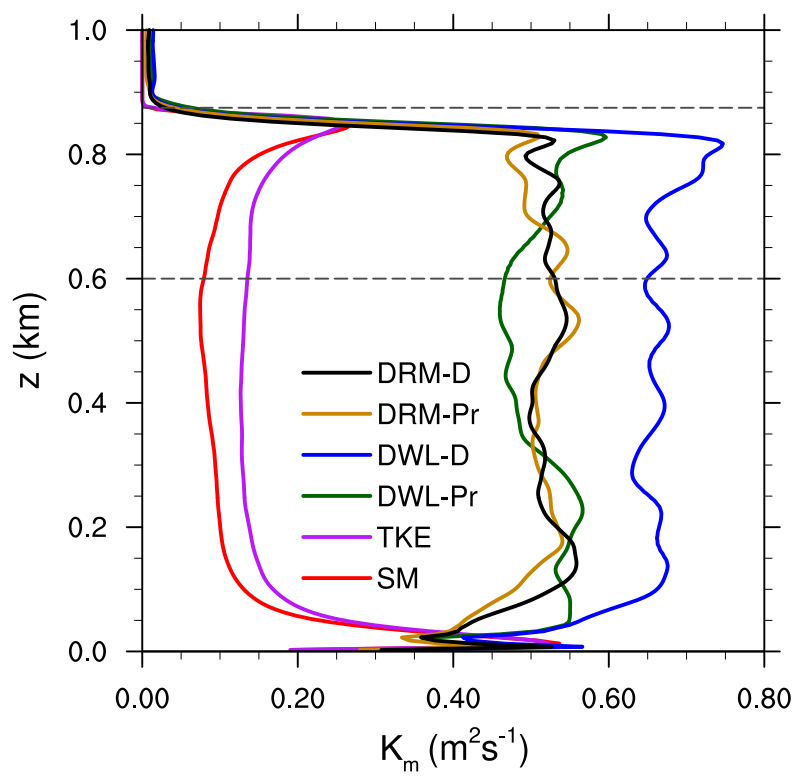

FIG. 3. Horizontal- and time-mean profiles of SFS eddy viscosity $K_{m}$ for various models over the fourth hour of the simulations. 
a)

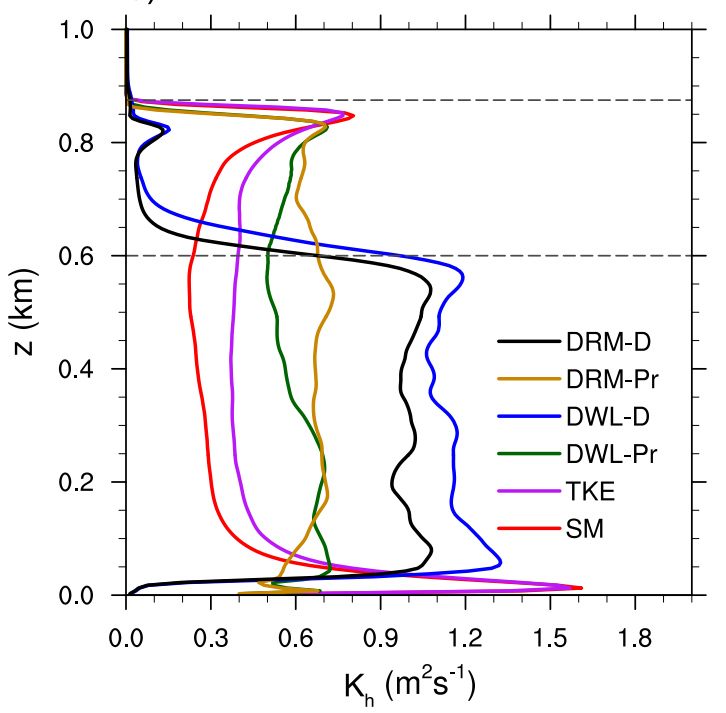

b)

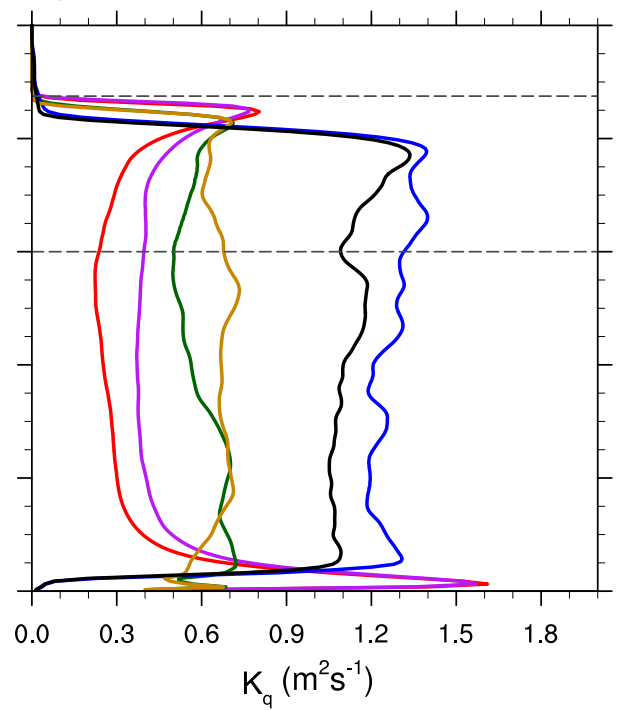

c)

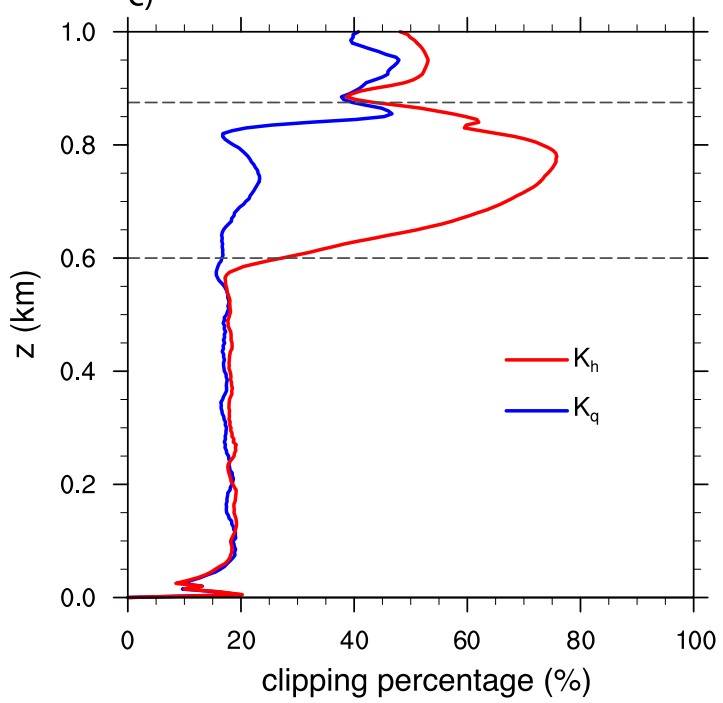

FIG. 4. Horizontal- and time-mean profiles of SFS eddy diffusivity for (a) potential temperature $K_{h}$ and (b) total water content $K_{q}$ for the turbulence models over the fourth hour of the simulations and (c) the percentage of grid cells in which $K_{h}$ and $K_{q}$ are clipped at zero over the fourth hour of the DWL-D simulation.

suppressed SFS mixing within the cloud. The other eddy diffusivity profiles are consistent with the pairing of turbulence models. DRM-Pr and DWL-Pr exhibit similar, intermediate values below the cloud and relatively large values above cloud base. SM and TKE have smaller values of $K_{h}$ below cloud than the other models and intermediate values within the cloud layer. Notably, the quality of the simulations appears to be more sensitive to the values of $K_{h}$ in the cloud than those in the subcloud layer. Taking DWL-D, DWL-Pr, and TKE, for example, the DWL-D simulation exhibits the most realistic cloud layer and turbulence statistics. While the $K_{h}$ values of DWL-Pr below the cloud base are closer to those of DWL-D than the $K_{h}$ values of TKE, the cloud in DWL-Pr is actually less realistic than that in the TKE simulation (cf. Fig. 1), which, within the cloud, exhibits $K_{h}$ values closer to those of DWL-D. This again underlines the importance of the cloud layer, where turbulence interacts with radiation (cf. Shi et al. 2018).

Figure $4 \mathrm{~b}$ shows the eddy diffusivities for total water content $K_{q}$. First, the distribution of diffusivities is consistent again with the pairing of turbulence models based on the selection of the Prandtl numberSM-TKE, DWL-Pr-DRM-Pr, and DWL-D-DRM-D. Second, only the dynamic models, DWL-D and DRM-D, exhibit different eddy diffusivities for heat and water. 
a)

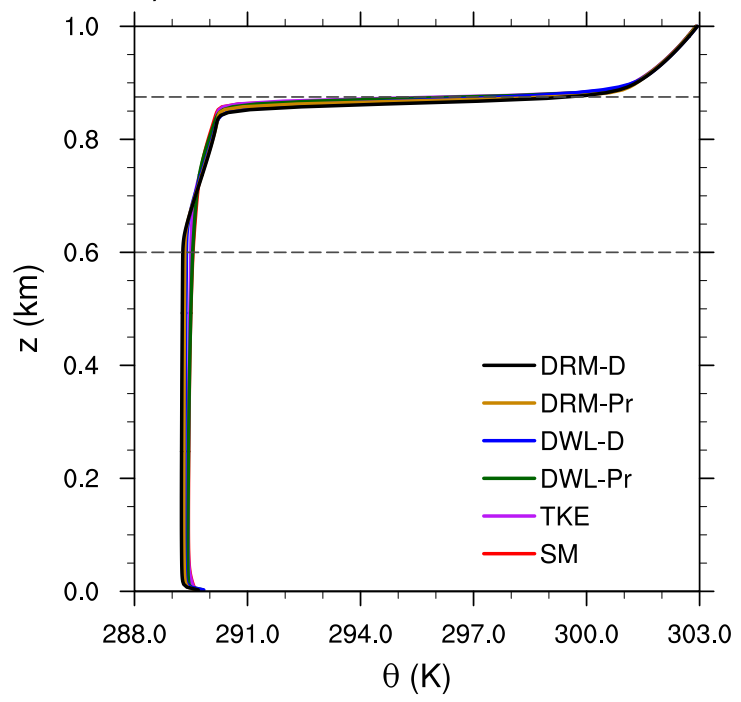

b)

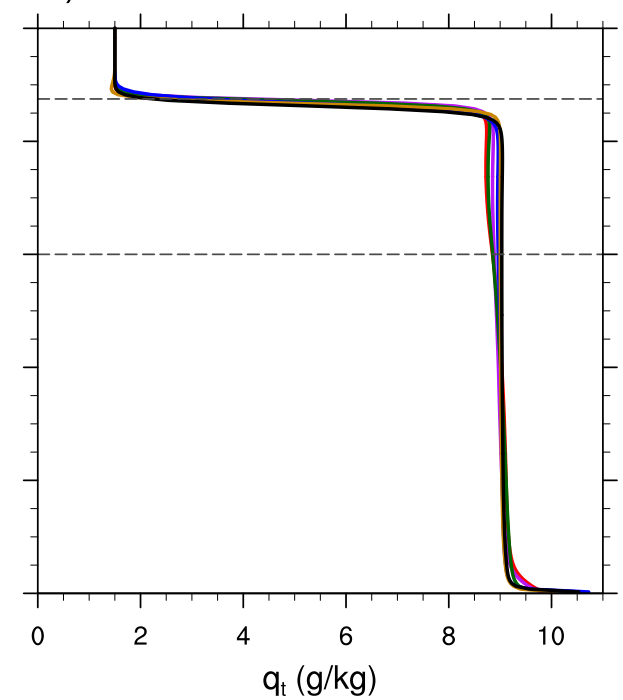

FIG. 5. Horizontal- and time-mean profiles of (a) potential temperature $\theta$ and (b) total water mixing ratio $q_{t}$ over the fourth hour of the simulations.

In DWL-D and DRM-D, $K_{q}$ and $K_{h}$ have similar values $\left(\sim 1.2 \mathrm{~m}^{2} \mathrm{~s}^{-1}\right)$ below cloud, but show drastically different values within the cloud, with $K_{h}$ being near zero and with $K_{q}$ being still close to $1.2 \mathrm{~m}^{2} \mathrm{~s}^{-1}$. Within the cloud layer, $K_{q}$ in the dynamic models becomes near zero only in a narrow region close to cloud top. The other models cannot produce different eddy diffusivities for water and heat because they rely on a single formulation for $\mathrm{Pr}$ and Sc, which are used to obtain $K_{q}$ and $K_{h}$ from eddy viscosity.

Why are the structures of $K_{h}$ and $K_{q}$ so different in the boundary layer for the dynamic models? A probable cause is that the transport of potential temperature in the cloud layer tends to be countergradient. In the boundary layer and the majority of the cloud, the total water mixing ratio is well mixed. Potential temperature is well mixed below the cloud but exhibits gradients within the cloud layer (Fig. 5). In the DWL-D simulation, $K_{h}$ is clipped at zero in about $60 \%$ of the grid cells between 600 and $900 \mathrm{~m}$, whereas $K_{q}$ is only clipped in about $24 \%$ of the grid cells in the same region (Fig. 4 c). The occurrence of clipping means that the dynamic procedure tends to predict negative eddy diffusivity, but that is not allowed (because of clipping) so as to prevent numerical instability. For the grid cells with positive $K_{h}$ values, the mixing due to the corresponding downward $\theta$ flux, which moves warm (positively buoyant) air downward and cold (negatively buoyant) air upward, is likely still an inefficient process. In contrast, the mixing of total water is likely not very different within and below the clouds; therefore, the magnitude of $K_{q}$ is similar throughout the boundary layer.

\section{Prandtl and Schmidt numbers}

By definition, $\operatorname{Pr}=K_{m} / K_{h}$ and Sc $=K_{m} / K_{q}$. Computing Pr and Sc at each grid point would lead to $K_{m}$ being divided by zero at some points; thus, we use the horizontal means of eddy viscosity and eddy diffusivities to calculate Pr and Sc. As a consequence, the variability of $\mathrm{Pr}$ and $\mathrm{Sc}$ is slightly reduced, but its dependence on height can still be reflected. As shown in Fig. 6, below the cloud layer $(z<600 \mathrm{~m})$, the values of Pr and Sc are essentially constant in the well-mixed zone. The TKE and $\mathrm{SM}$ values are specified in the CM1 code as $1 / 3$. The DWL-Pr and DRM-Pr models have a value of 0.7 [assigned to neutral conditions in (1)]. The dynamic models, whose performance against the field data is generally the best, suggest that the appropriate neutral case value is about 0.5 .

In the cloud, $\mathrm{Pr}$ and Sc behave quite differently for each model, Again, the TKE and SM values are almost constant at 1/3. DWL-Pr and DRM-Pr models remain fairly constant until just at cloud top, where they increase rapidly because of the prescribed dependence of Pr on stratification. DWL-D and DRM-D behave alike, but the actual Pr and Sc profiles are distinct in the cloud layer, showing the difference in the physics of heat and water content. For DWL-D and DRM-D, Pr is on the order of 10 for most levels in the cloud, while Sc reaches the order of 10 only in a narrow region below cloud top. This behavior appears to be an essential factor in the proper representation of the physics and is reflected in the excellent match between simulation and field data shown earlier. The profiles above the 
a)

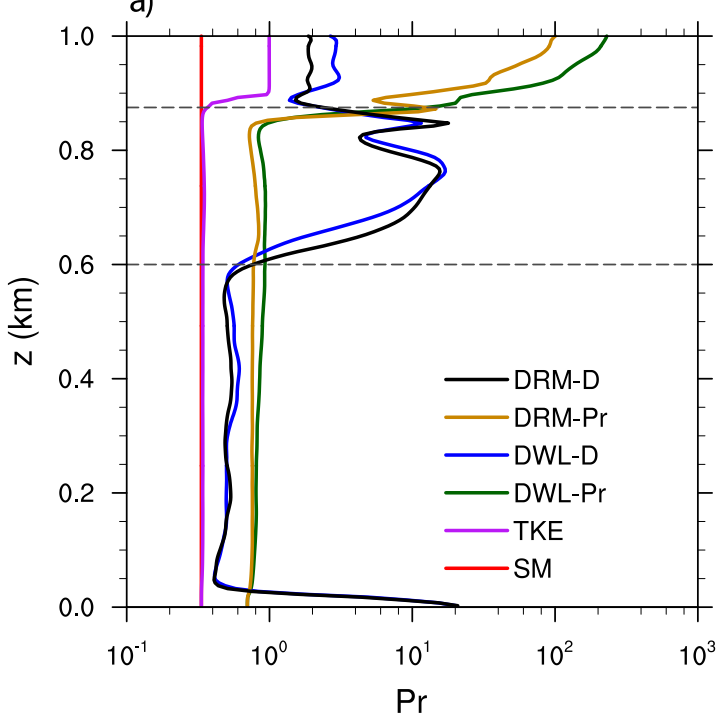

b)

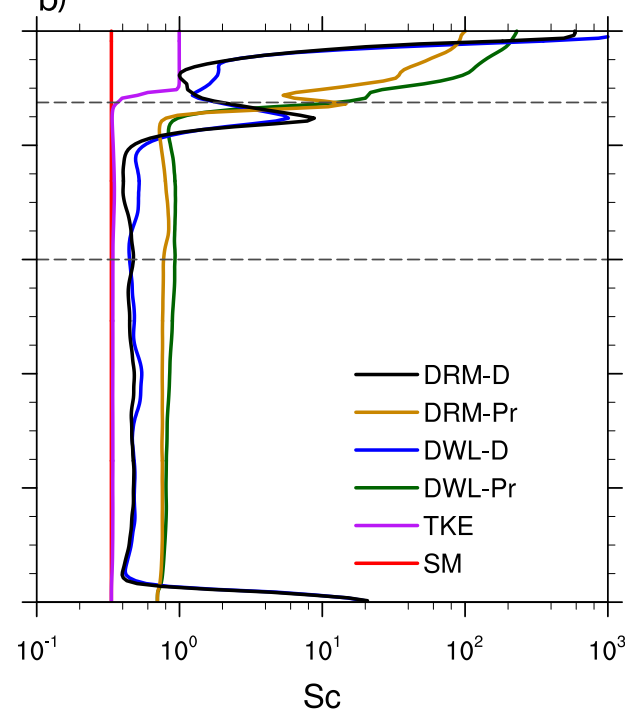

FIG. 6. Horizontal- and time-mean profiles of (a) SFS Pr for potential temperature and (b) SFS Sc for total water content for the turbulence models over the fourth hour of the simulations.

cloud top are not significant because the flow is not turbulent there and has little influence on the dynamics of the boundary layer.

\section{Summary and conclusions}

In this study, we investigate the critical role of the turbulence model, specifically in determining scalar mixing and the associated Prandtl and Schmidt numbers, in successful simulation of the stratocumuluscapped boundary layer. Of the six turbulence models studied, the schemes using a dynamic eddy diffusivity or reconstruction approach (DWL-D, DRM-D, and DRM-Pr) produce simulations that match observation well, whereas other models (SM, TKE, and DWL-Pr) produce an unrealistically thin cloud and a decoupled boundary layer. Notably, DWL-Pr's performance is inferior, suggesting that using a dynamic procedure (without reconstruction) only for momentum and relying on an empirical SFS Prandtl number for eddy diffusivities is insufficient. The schemes with good performance exhibit small values of SFS eddy diffusivities for heat and water in the cloud and/or near cloud top, suppressing mixing in those regions. The reconstructed fluxes can alter the total SFS flux by adding the reconstructed RSFS components [see (2) and (3)]. These are key factors that guaranteed the good quality of simulations in DWL-D, DRM-D, and DRM-Pr.

Traditional turbulence models fail to account for varying SFS Prandtl and Schmidt numbers in the flow. In the SM and TKE model, SGS Prandtl and Schmidt numbers are typically set between $1 / 3$ and 1 in atmospheric modeling. In contrast, the effective SGS Prandtl number in dynamic models is found here to be about 0.5 below cloud and on the order of 10 within the cloud. This large value in the cloud minimizes turbulent mixing of the potential temperature field. The SFS Schmidt number in the dynamic models is found to be about 0.5 at most levels of the boundary layer and becomes on the order of 10 only at levels close to but below cloud top. The complex behaviors of the SFS Prandtl and Schmidt numbers is not captured by the empirical formulation of Venayagamoorthy and Stretch (2010) either, as seen by the DWL-Pr results.

Determining Pr and Sc to be on the order of 10 may seem somewhat large for nearly neutral conditions. The Pr and Sc values used in LES codes, however, are related to SFS turbulence instead of to the full-spectrum turbulence in the boundary layer; thus, these values may be different from the Pr and Sc for the turbulence at resolved and larger scales. Furthermore, it is known that the dependence of $\operatorname{Pr}$ on stability is complicated; for given stability, $\operatorname{Pr}$ can exhibit substantial variability in observation data (e.g., Grachev et al. 2007).

The convention of using $\operatorname{Pr}=1 / 3$ in LES may originate from Deardorff (1971), who determined the value $1 / 3$ by comparing spectra of kinetic energy and potential temperature variance in simulations. Other values of Pr ranging from 0.3 to 1 were also used previously in different LES cases (e.g., Grötzbach and Schumann 1979; Schmidt and Schumann 1989; Lesieur and Metais 1996; Canuto and Cheng 1997; Stevens et al. 1999). 
In Stevens et al. (2005), however, the model that produces the best results for the SCBL is a code in which SFS mixing of scalars is turned completely off. The mixing is then represented by the physics of resolvedscale transport terms and their implied truncation error. Thus, the effective SFS Pr and Sc numbers implicitly depend on the properties of the advection schemes. We also conducted a special simulation using the Smagorinsky model and with Pr set at 10 . This special run did exhibit significant improvement in the simulation results with more realistic cloud and turbulence statistics (not shown for brevity).

It is clearly difficult to know what the SFS Pr and Sc in a flow should be a priori. Thus, models relying on specified numbers can fail if the specification does not match the physics. Dynamic models, with or without reconstruction, allow the computation of SFS scalar fluxes without the specification of a Prandtl or Schmidt number. Given the success shown here in the SCBL case, dynamic approaches appear to be promising tools to capture flow physics and provide scalar transport simulations with higher fidelity.

Acknowledgments. The authors thank three anonymous reviewers for their helpful reviews. This research was supported by National Science Foundation (NSF) Grants AGS-1503860 and AGS-1503885. We acknowledge high-performance computing support from Yellowstone (ark:/85065/d7wd3xhc) provided by NCAR's Computational and Information Systems Laboratory, which is sponsored by NSF.

\section{REFERENCES}

Bardina, J., J. H. Ferziger, and W. C. Reynolds, 1983: Improved turbulence models based on large eddy simulation of homogeneous, incompressible, turbulent flows. Stanford University Dept. of Mechanical Engineering Tech. Rep. TF-19, 174 pp.

Borges, R., M. Carmona, B. Costa, and W. S. Don, 2008: An improved weighted essentially non-oscillatory scheme for hyperbolic conservation laws. J. Comput. Phys., 227, 3191-3211, https://doi.org/10.1016/j.jcp.2007.11.038.

Bryan, G. H., and J. M. Fritsch, 2002: A benchmark simulation for moist nonhydrostatic numerical models. Mon. Wea. Rev., 130, 2917-2928, https://doi.org/10.1175/1520-0493(2002)130<2917: ABSFMN $>2.0 . \mathrm{CO} ; 2$.

— and R. Rotunno, 2009: The maximum intensity of tropical cyclones in axisymmetric numerical model simulations. Mon. Wea. Rev., 137, 1770-1789, https://doi.org/10.1175/2008MWR2709.1.

Canuto, V., and Y. Cheng, 1997: Determination of the Smagorinsky-Lilly constant $C_{S}$. Phys. Fluids, 9, 1368-1378, https://doi.org/10.1063/1.869251.

Chow, F. K., R. L. Street, M. Xue, and J. H. Ferziger, 2005: Explicit filtering and reconstruction turbulence modeling for largeeddy simulation of neutral boundary layer flow. J. Atmos. Sci., 62, 2058-2077, https://doi.org/10.1175/JAS3456.1.
Deardorff, J. W., 1971: On the magnitude of the subgrid scale eddy coefficient. J. Comput. Phys., 7, 120-133, https://doi.org/ 10.1016/0021-9991(71)90053-2.

— 1980: Stratocumulus-capped mixed layers derived from a three-dimensional model. Bound.-Layer Meteor., 18, 495-527, https://doi.org/10.1007/BF00119502.

Findikakis, A. N., and R. L. Street, 1979: An algebraic model for subgrid-scale turbulence in stratified flows. J. Atmos. Sci., 36, 1934-1949, https://doi.org/10.1175/1520-0469(1979)036<1934: AAMFSS $>2.0 . \mathrm{CO} ; 2$.

Goldberg, U. C., S. Palaniswamy, P. Batten, and V. Gupta, 2010: Variable turbulent Schmidt and Prandtl number modeling. Eng. Appl. Comput. Fluid Mech., 4, 511-520, https://doi.org/ 10.1080/19942060.2010.11015337.

Grachev, A. A., E. L Andreas, C. W. Fairall, P. S. Guest, and P. O. G. Persson, 2007: On the turbulent Prandtl number in the stable atmospheric boundary layer. Bound.-Layer Meteor. 125, 329-341, https://doi.org/10.1007/s10546-007-9192-7.

Grötzbach, G., and U. Schumann, 1979: Direct numerical simulation of turbulent velocity, pressure, and temperature fields in channel flows. Turbulent Shear Flows I, Springer, 370-385, https://doi.org/10.1007/978-3-642-46395-2_28.

Kirkpatrick, M. P., A. S. Ackerman, D. E. Stevens, and N. N. Mansour, 2006: On the application of the dynamic Smagorinsky model to large-eddy simulations of the cloud-topped atmospheric boundary layer. J. Atmos. Sci., 63, 526-546, https://doi.org/10.1175/JAS3651.1.

Lesieur, M., and O. Metais, 1996: New trends in large-eddy simulations of turbulence. Annu. Rev. Fluid Mech., 28, 45-82, https://doi.org/10.1146/annurev.fl.28.010196.000401.

Matheou, G., and D. Chung, 2014: Large-eddy simulation of stratified turbulence. Part II: Application of the stretchedvortex model to the atmospheric boundary layer. J. Atmos. Sci., 71, 4439-4460, https://doi.org/10.1175/JAS-D-13-0306.1.

Moeng, C.-H., 2000: Entrainment rate, cloud fraction, and liquid water path of PBL stratocumulus clouds. J. Atmos. Sci., 57, 3627-3643, https://doi.org/10.1175/1520-0469(2000)057<3627: ERCFAL $>2.0 . \mathrm{CO} ; 2$.

Perot, J. B., and J. Gadebusch, 2009: A stress transport equation model for simulating turbulence at any mesh resolution. Theor. Comput. Fluid Dyn., 23, 271-286, https://doi.org/ 10.1007/s00162-009-0113-x.

Pressel, K. G., S. Mishra, T. Schneider, C. M. Kaul, and Z. Tan, 2017: Numerics and subgrid-scale modeling in large eddy simulations of stratocumulus clouds. J. Adv. Model. Earth Syst., 9, 1342-1365, https://doi.org/10.1002/2016MS000778.

Rasam, A., G. Brethouwer, and A. V. Johansson, 2013: An explicit algebraic model for the subgrid-scale passive scalar flux. J. Fluid Mech., 721, 541-577, https://doi.org/10.1017/jfm.2013.81.

Schmidt, H., and U. Schumann, 1989: Coherent structure of the convective boundary layer derived from large-eddy simulations. J. Fluid Mech., 200, 511-562, https://doi.org/10.1017/ S0022112089000753.

Shi, X., H. L. Hagen, F. K. Chow, G. H. Bryan, and R. L. Street, 2018: Large-eddy simulation of the stratocumulus-capped boundary layer with explicit filtering and reconstruction turbulence modeling. J. Atmos. Sci., 75, 611-637, https://doi.org/ 10.1175/JAS-D-17-0162.1.

Stevens, B., C.-H. Moeng, and P. P. Sullivan, 1999: Large-eddy simulations of radiatively driven convection: Sensitivities to the representation of small scales. J. Atmos. Sci., 56, 3963-3984, https://doi.org/10.1175/1520-0469(1999)056<3963: LESORD $>2.0 . \mathrm{CO} ; 2$. 
- and Coauthors, 2005: Evaluation of large-eddy simulations via observations of nocturnal marine stratocumulus. Mon. Wea Rev., 133, 1443-1462, https://doi.org/10.1175/MWR2930.1.

Vanzanten, M. C., P. G. Duynkerke, and J. W. M. Cuijpers, 1999: Entrainment parameterization in convective boundary layers. J. Atmos. Sci., 56, 813-828, https://doi.org/10.1175/1520-0469 (1999)056<0813:EPICBL>2.0.CO;2.

Venayagamoorthy, S. K., and D. D. Stretch, 2010: On the turbulent Prandtl number in homogeneous stably stratified turbulence. J. Fluid Mech., 644, 359-369, https://doi.org/ 10.1017/S002211200999293X.

Wilson, J. M., and S. K. Venayagamoorthy, 2015: A shear-based parameterization of turbulent mixing in the stable atmospheric boundary layer. J. Atmos. Sci., 72, 1713-1726, https:// doi.org/10.1175/JAS-D-14-0241.1.

Wong, V. C., and D. K. Lilly, 1994: A comparison of two dynamic subgrid closure methods for turbulent thermal convection. Phys. Fluids, 6, 1016-1023, https://doi.org/10.1063/1.868335. 